ORIGINAL

\author{
J.S. Schauberger \\ P.G. Kranz \\ K.R. Choudhury \\ J.D. Eastwood \\ L. Gray \\ J.K. Hoang
} RESEARCH

\section{CT-Guided Lumbar Nerve Root Injections: Are We Using the Correct Radiation Dose Settings?}

BACKGROUND AND PURPOSE: Selecting a lower tube current for CT fluoroscopic spine injections is a method of radiation dose reduction. Ideally tube current should be tailored to the patient's body habitus, but a greater influence on tube current may be the proceduralist's personal preference. The purpose of this study was to compare tube current and fluoroscopy time of different proceduralists for lumbar spine CT-guided selective nerve root blocks, and to correlate image quality to patient diameter and tube current.

MATERIALS AND METHODS: Eighty CT-guided SNRBs performed by 4 proceduralists were retrospectively reviewed for tube current and fluoroscopy time. Patient body habitus was evaluated by measuring anteroposterior diameters on scout images. Image quality was evaluated objectively and subjectively: noise was measured in the psoas muscle and images were graded on a 3-point scale.

RESULTS: The mean tube current was $59 \pm 20 \mathrm{~mA}$ and mean fluoroscopy time was $10.4 \pm 7.5$ seconds. The mean tube current between proceduralists differed by almost 2-fold, and there was greater than 2-fold difference in mean fluoroscopy time $(P<.0001$ and .01 , respectively). Mean AP body size was $27 \pm 5 \mathrm{~cm}$. When categoric data of tube current and AP diameter were analyzed, only AP diameter was a statistically significant variable influencing image noise $(P=.009)$. Twenty of 23 patients with AP diameter $\leq 30 \mathrm{~cm}$ had adequate to excellent image quality, even with lower tube current of $\leq 40 \mathrm{~mA}$.

CONCLUSIONS: Wide variability in tube current selection between proceduralists calls for a more objective method of selecting tube current to minimize radiation dose. Body size, measured by AP diameter, had the greatest influence on image quality. This could be used to identify patients for lower tube current selection.

ABBREVIATIONS: $\mathrm{AP}=$ anteroposterior $; \mathrm{CTDI}_{\mathrm{vol}}=\mathrm{CT}$ dose index volume; $\mathrm{SNRB}=$ selective nerve root block

$\mathbf{T}$ he use of CT guidance for spine steroid injections has increased with greater availability and advances of CT fluoroscopy. In the lumbar spine, CT guidance offers advantages over conventional fluoroscopy of improved soft tissue resolution, ${ }^{1}$ improved precision in needle placement, ${ }^{2,3}$ and decreased procedure time. ${ }^{4,5}$ However, because of the higher tube current for CT compared with conventional fluoroscopy, when possible, proceduralists need to be mindful of radiation dose optimization and minimize dose.

Key determinants of radiation dose in CT fluoroscopy are tube current and fluoroscopy time; both parameters are linearly proportional to radiation dose. While fluoroscopy time varies depending on the difficulty of the procedure and experience of the proceduralist, tube current is selected before the procedure. Thus, selecting a lower fluoroscopy tube current is a simple and effective method of reducing radiation dose. In some practices, tube current for CT fluoroscopic procedures is set as a default $\mathrm{mA}$, but at many centers, the proceduralist assumes an active role in selecting the tube current. The pro-

Received December 5, 2011; accepted after revision February 3, 2012.

From the Departments of Radiology (J.S.S., P.G.K., K.R.C., J.D.E., L.G., J.K.H.) and Radiation Oncology (J.K.H.), Duke University Medical Center, Durham, North Carolina.

Previously presented in part at: Annual Meeting of the American Society of Neuroradiology, June 6, 2011; Seattle, Washington.

Please address correspondence to Jenny K. Hoang, Duke University Medical Center, Department of Radiology, Box 3808, Erwin Rd, Durham, NC 27710; e-mail: jennykh@ gmail.com

http://dx.doi.org/10.3174/ajnr.A3096 ceduralist's choice of tube current for lumbar spine procedures may be partly based on the patient's body habitus, but a large component of the variability may also be due to the proceduralist's personal preference. As a result, there is wide variability in the reported tube current settings for lumbar spine CT fluoroscopy procedures. ${ }^{3-7}$

The aim of this study was to compare the tube current selection and fluoroscopy time of different proceduralists for lumbar spine SNRB in our practice. We sought to learn the variability in tube current to help us form strategies for optimizing CT fluoroscopy utility, while keeping radiation dose as low as reasonably achievable. A secondary aim was to use the cohort of patients to study the relationship between image quality, tube current, and body habitus.

\section{Materials and Methods}

\section{Patient Population}

We selected 4 procedural neuroradiologists with varying years of experience $(2,8,15$, and 15 years) and retrospectively reviewed 20 consecutive SNRB procedures for each of the neuroradiologists. The procedures were all performed at an academic institution under CT guidance from December 2008 to October 2010. Patients having multiple injections were excluded, as additional procedures would lengthen the fluoroscopy time and affect the choice of tube current (eg, higher tube current for central epidural injections). The study was approved by our institutional review board and was compliant with the Health Insurance Portability and Accountability Act. 

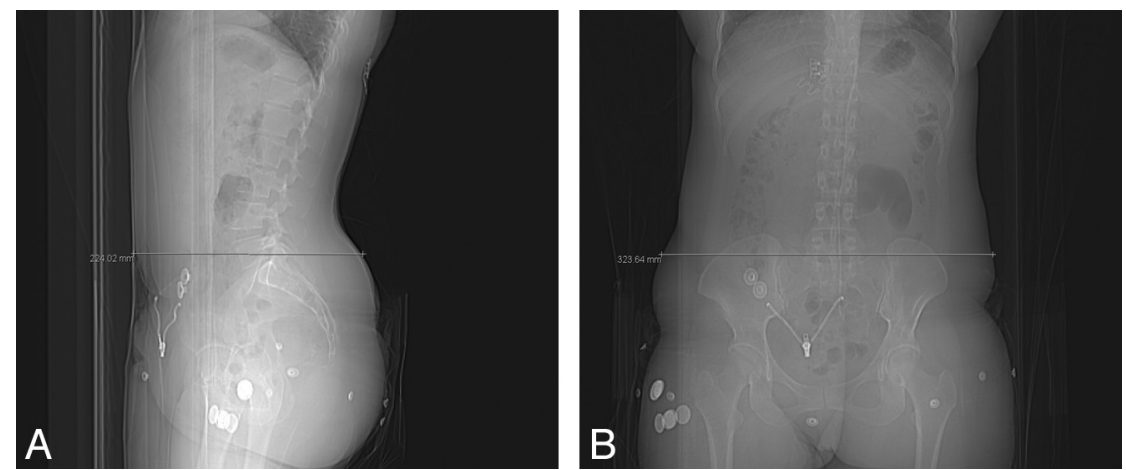

Fig 1. Measurement of body diameter for a 51-year-old man having an L5 SNRB. A, AP diameter was measured from the lateral scout radiograph at the level of the exiting L5 nerve root. $B$, Transverse diameter was measured from the frontal scout radiograph at the level of the exiting $L 5$ nerve root.
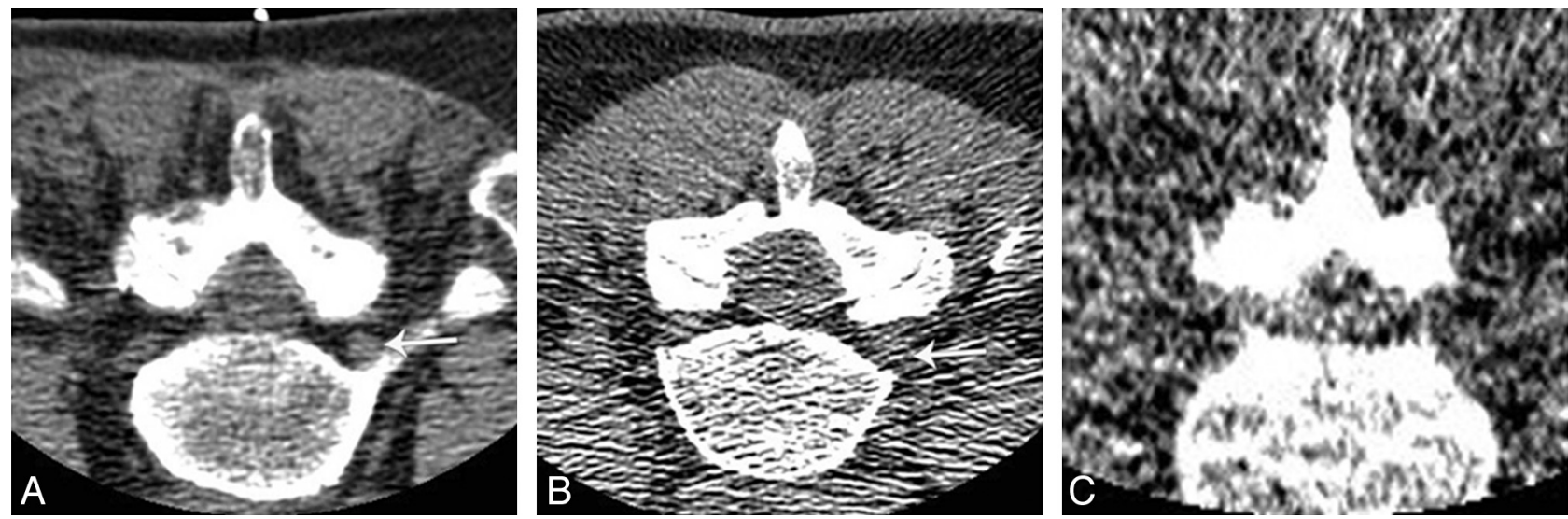

Fig 2. Image analysis score system. $A$, Grade II represented cases in which the exiting nerve root (arrow) was well delineated and confidently identified. $B$, Grade I represented cases in which the nerve root (arrow) was identified, but not confidently, and required image window and level manipulation or review of the planning $\mathrm{CT}$ images. $C$, Grade 0 represented cases in which the nerve root could not be identified.

\section{CT Protocol and SNRB Procedure}

All procedures were performed on a 16-section multidetector CT scanner (LightSpeed 16; GE Healthcare, Milwaukee, Wisconsin). Initial scout images (scanograms) were obtained in the lateral and frontal projections, followed by a planning CT for evaluation of extent of degenerative changes and for planning the needle placement. The CT parameters for the planning CT were the same as our standard clinical protocol for lumbar spine CT: $120 \mathrm{kVp}$, automatic tube current modulation with Smart $\mathrm{mA}$ (minimum $100 \mathrm{~mA}$, maximum $440 \mathrm{~mA}$, noise index $=10$ ), contiguous section thickness $2.5 \mathrm{~mm}$, and $25 \mathrm{~mm}$ field of view. The area scanned ( $\mathrm{z}$-axis) varied depending on available prior imaging and proceduralist preference.

Following the planning CT, the procedural neuroradiologist selected a fixed tube current for the CT fluoroscopy procedure. The technique for performing CT fluoroscopy-guided SNRB has been previously described in the literature. ${ }^{4,6,8}$ Three CT images were obtained each time the radiologist stepped on the CT fluoroscopy pedal and each image was equivalent to 0.33 seconds of exposure time (total 1 second).

\section{Radiation Parameters and Body Size Assessment}

Data collected from each procedure included the patient's age, sex, and level and side of nerve root injection. The fluoroscopy tube current ( $\mathrm{mA}$ ), fluoroscopy time (seconds), and $\mathrm{CTDI}_{\mathrm{vol}}$ of the CT fluoroscopy portion of the examination were obtained from the CT dose report.

To determine whether there was any relationship between patient body size and tube current, we evaluated habitus by measuring the AP and transverse diameter on the scout images (Fig 1). Measurements were taken from skin-to-skin surface, perpendicular to the CT bed, on the lateral and frontal scout radiographs, respectively, at the level of the nerve root being treated.

\section{Image Quality Assessment}

Images were analyzed on a PACS (Centricity, Version 2, GE Healthcare) workstation and assessed by 2 methods. First, image noise was evaluated on the axial fluoroscopy images at the level of the nerve root being treated by placing $20-\mathrm{mm}^{2}$ circular ROIs and measuring the standard deviation of attenuation in Hounsfield units (a measure of image noise ${ }^{9}$ ) in the psoas muscle. Three measurements of psoas noise were used to obtain a mean value for statistical analysis. Patients who did not have a visible psoas muscle were excluded from image analysis.

The second measure of image quality was subjective. Nerve root visibility was first assessed independently by 2 fellowship-trained neuroradiologists with 7 and 8 years of experience reading lumbar spine CT scans. The readers were blinded to the proceduralists who performed the SNRBs. The readers graded image quality on a 3-point scale of 0 (worst) to II (best) for the higher tube current pretreatment planning scan and the CT fluoroscopy images (Fig 2). Grade II represented cases in which the exiting nerve root was well delineated and confidently identified. Grade I represented cases in which the nerve root was identified, but not confidently, requiring image window and level manipulation or review of the planning CT images. Grade 0 
Table 1: Tube current, fluoroscopy time, patient AP diameter, and CTDI $_{\text {vol }}$ for the 4 proceduralists

\begin{tabular}{lccccc}
\hline & A & B & C & D & $P$ Value \\
\hline Tube current (mA) & $66(7)$ & $42(11)$ & $49(17)$ & $80(16)$ & $<.0001$ \\
Fluoroscopy time (seconds) & $10(5)$ & $6(5)$ & $12(7)$ & $14(10)$ & .01 \\
AP diameter (cm) & $26(5)$ & $28(7)$ & $27(3)$ & $29(5)$ & .27 \\
CTDI ${ }_{\text {vol }}$ of CT fluoroscopy & $88(50)$ & $34(25)$ & $78(54)$ & $149(113)$ & $<.0001$ \\
$\quad$ & & & & \\
(mGy) & & &
\end{tabular}

represented cases in which the nerve root could not be identified. The neuroradiologists first graded image quality independently. For each case in which there was a disagreement, a consensus opinion was reached. We excluded cases in which the nerve root was not seen on higher radiation dose planning CT (Grade 0 ), as it would be unlikely that the nerve would be visible on the lower-dose CT fluoroscopy images.

\section{Statistical Analysis}

The data were entered into an Excel spreadsheet (2007 version, Microsoft, Redmond, Washington). Statistical analyses were performed using SAS Enterprise (Version 4.2, SAS Institute, Cary, North Carolina). Body size and tube current were analyzed as continuous variables and categoric data. Body size and tube current were categorized based on their means and standard deviations.

ANOVA was used to evaluate for differences in treatment tube current, fluoroscopy time, and patient diameter between the 4 proceduralists. The relationship of image quality relative to patient diameter and tube current was evaluated by multiple linear regression models. The relationship of image grade relative to categoric data for tube current and patient size was evaluated by the $\chi^{2}$ test. A 2 -tailed $P$ value of less than .05 was considered to be statistically significant.

\section{Results}

\section{Patient Population and Body Size}

In 80 subjects, the mean age was 60 years (range 17-88) and 50 patients were female. Levels treated were from the first lumbar to first sacral nerve root (L1-S1), but most commonly L4 (28\%) and L5 (46\%).

The mean transverse body size was $37 \pm 5 \mathrm{~cm}(\mathrm{SD})$ and the mean AP body size was $27 \pm 5 \mathrm{~cm}$. There was no statistically significant difference in the transverse diameter or AP diameter of patients among the 4 proceduralists $(P \geq .27)$ (Table 1$)$. Because AP and transverse diameters were strongly correlated $(r=0.84)$, only AP diameter was selected for correlation with image quality. AP diameter was divided into small $(<20 \mathrm{~cm})$, medium $(20-30 \mathrm{~cm})$, and large $(>30 \mathrm{~cm})$ body size based on the mean and standard deviation (mean $27 \mathrm{~cm}$ rounded to $25 \mathrm{~cm}, \pm 5 \mathrm{SD})$.

\section{Tube Current and Fluoroscopy Time}

The mean tube current used was $59 \pm 20 \mathrm{~mA}$ (range 20-120) and the mean fluoroscopy time was $10.4 \pm 7.5$ seconds (range 2.3-38). Tube current was categorized into 3 groups: low $(\leq 40)$, medium $(40-80)$, and high $(>80) \mathrm{mA}$. Table 1 shows the tube current and fluoroscopy time for the 4 proceduralists. There were statistically significant differences between both the tube current and fluoroscopy time between the proceduralists $(P<.0001$ and .01 , respectively). Proceduralist $\mathrm{B}$, who

\begin{tabular}{lccc}
\hline \multicolumn{4}{c}{ Table 2: Image noise by tube current and body AP diameter } \\
\hline & Number & Mean Image Noise (HU) & $P$ Value \\
\hline Tube current (mA) & & & .25 \\
Low ( $\leq 40)$ & 25 & $84(38)$ & \\
Medium (40-80) & 35 & $69(41)$ & \\
High ( $>80)$ & 17 & $69(25)$ & .009 \\
AP diameter (cm) & & & \\
Large (>30) & 17 & $92(43)$ & \\
Medium (20-30) & 56 & $71(34)$ & \\
Small $(<20)$ & 4 & $33(11)$ & \\
\hline
\end{tabular}

Note:-ANOVA was used to evaluate differences in image noise in categories of tube current and body AP diameter. Numbers in parentheses are standard deviations. HU indicates Hounsfield unit.

\begin{tabular}{|c|c|c|c|c|}
\hline & \multicolumn{3}{|c|}{ Image Quality Score } & \multirow[b]{2}{*}{$P$ Value } \\
\hline & 0 & I & II & \\
\hline Tube current (mA) & & & & .76 \\
\hline Low $(\leq 40)$ & $6(23)$ & $4(15)$ & $16(62)$ & \\
\hline Medium (40-80) & $4(12)$ & $8(25)$ & $20(63)$ & \\
\hline High $(>80)$ & $2(12)$ & $4(25)$ & $10(63)$ & \\
\hline AP diameter $(\mathrm{cm})$ & & & & .001 \\
\hline Large $(>30)$ & $7(39)$ & $7(39)$ & $4(22)$ & \\
\hline Medium (20-30) & $5(10)$ & $9(17)$ & $38(73)$ & \\
\hline Small $(<20)$ & 0 & 0 & $4(100)$ & \\
\hline
\end{tabular}

Note:-The $\chi^{2}$ test was used to evaluate differences in image quality score in categories of tube current and body AP diameter. Numbers in parentheses are row percentages.

had 15 years of experience, had both the lowest mean tube current selection ( $42 \mathrm{~mA}$ ) and the shortest mean fluoroscopy time (6 seconds). Proceduralist D, who also had 15 years of experience, had both the highest mean tube current selection (80 $\mathrm{mA})$ and the longest mean fluoroscopy time (14 seconds).

\section{Image Quality}

Three patients were excluded from psoas muscle noise measurement because the muscle was not visible on the fluoroscopic images. The mean psoas noise in 77 patients was $74 \pm$ $37 \mathrm{HU}$. Table 2 shows image noise by categoric groups of tube current and AP diameter. Noise increased with increasing body size categories and with the lowest tube current category $(\leq 40 \mathrm{~mA})$. When categoric data of tube current and AP diameter were analyzed, only AP diameter was a statistically significant variable influencing image noise $(P=.009)$. By multiple linear regression, both tube current and AP diameter were statistically significant independent determinants of image noise $(r=0.60, P<.001)$.

Six cases were excluded for assessment of image quality grade on fluoroscopy images because the nerve root was not visualized on the planning CT due to adjacent foraminal degenerative changes. In the remaining 74 patients, grades $0, \mathrm{I}$, and II image quality were seen in 12,16 , and 46 patients, respectively. Table 3 shows image grade by tube current and AP diameter.

The nerve root was identified (grade I or II) in 51 of 56 (91\%) patients with AP diameter $\leq 30 \mathrm{~cm}$. For patients with AP diameter $>30 \mathrm{~cm}$ in size, the nerve root was identified in only 11 of 18 cases $(61 \%)$. The $\chi^{2}$ test found AP body diameter, and not tube current, to be a statistically significant determinant of image quality $(P=.001)$ (Table 3$)$. By linear regression, both tube current and AP diameter were statistically sig- 
nificant independent determinants of image noise $(P=.045$ and $<.001$, respectively).

Of 12 patients who had grade 0 image quality, 7 (58\%) had AP diameters $>30 \mathrm{~cm}$ (large body size) and $5(42 \%)$ had AP diameter between $20-30 \mathrm{~cm}$. In the 5 cases in which the nerve root could not be identified in patients with AP diameter $\leq 30$ $\mathrm{cm}$, the average CT fluoroscopy tube current was $46 \mathrm{~mA}$. One of the 5 patients had extensive spinal hardware, limiting nerve root visibility. Three others had little fat around the nerve root, making visual separation of the nerve root from the paraspinal and psoas muscles difficult. In the fifth case, the tube current was $30 \mathrm{~mA}$ and noise was the limiting factor in nerve root identification.

No patient with AP diameter $<20 \mathrm{~cm}$ had grade 0 image quality. When low tube current $(\leq 40 \mathrm{~mA})$ was used in patients with AP diameter of $30 \mathrm{~cm}$ or less, 20/23 (87\%) had grade I or II image quality.

\section{Discussion}

Lowering the tube current for CT fluoroscopy can be a simple and effective method of reducing radiation exposure to both the patient and proceduralist. Currently, CT scanners will allow tube current selection from as low as 10 to above $200 \mathrm{~mA}$, and there are no specific vendor or regulatory recommendations for lumbar spine CT fluoroscopy procedures. In our study, there was a wide range of tube current selection among proceduralists independent of fluoroscopy time and body habitus. We found that lower tube currents $(\leq 40 \mathrm{~mA})$ still resulted in adequate image quality and that the major factor influencing image quality was body habitus.

Zhou et $\mathrm{al}^{10}$ retrospectively studied the variability of fluoroscopy time for conventional fluoroscopic-guided spine injection procedures and found the exposure time to vary greatly between physicians in different practice settings and within the same practices. In this study, there was a greater than 4 -fold difference in mean fluoroscopy time for epidural steroid injections between 2 physicians. Our study evaluated CTguided SNRB and also showed wide variation in mean fluoroscopy time between proceduralists (greater than 2 -fold) and almost 2 -fold differences in mean tube current selection. Because fluoroscopy time and tube current are linearly proportional to radiation dose, this translates to a 2 - to 4 -fold difference in radiation dose to a given patient, depending on their proceduralist. The differences could not be explained by differences in body habitus of the patient populations among the proceduralists. Given that the neuroradiologist with the shortest fluoroscopy time also had lower tube current selection, the most likely explanation for these differences in procedural time is heightened awareness of dose. Furthermore, reductions in tube current did not necessarily lead to a more difficult procedure and longer imaging times.

Because lowering tube current would lead to noisier images, we sought to understand the relationship between image quality, tube current, and body habitus. In our study, the greatest impact on subjective and objective image quality was the patient's body size. A cutoff AP diameter of $\leq 30 \mathrm{~cm}$ was associated with adequate image quality even at low tube current ranges $(\leq 40 \mathrm{~mA})$. Patients in whom this low-dose technique may be more challenging are those with spinal hardware in the field of view and patients with minimal fat around the nerve root on the planning CT. In clinical practice, this method of measuring the AP diameter on the scout image could potentially be used to identify patients for lower dose fluoroscopy for CT spine procedures, including patients having spine biopsies and other therapeutic steroid injections. The concept of tailoring CT dose settings to patient size is not novel. Body-size-specific protocols are currently used for pediatric diagnostic CT imaging. ${ }^{11,12}$ Although body weight is a more frequent measure of body size, several researchers favor the use of cross-sectional diameter, in a similar manner to our study, because it correlates with the distance of the pathway traversed by the $\mathrm{x}$-ray beam, and is also easy to measure on the scout image before the procedure. ${ }^{13,14}$

After identifying patients of smaller body habitus, the next clinically relevant step is to prescribe the most optimal low tube current. The lowest absolute tube current in our cohort of 80 patients was $20 \mathrm{~mA}$ and the lowest mean tube current by a proceduralist in our study was $42 \mathrm{~mA}$. Shepherd et al ${ }^{7}$ report even lower mean tube current of $12 \mathrm{~mA}$ after actively modifying the CT spine injection protocols at their institution. These authors rely on bony landmarks rather than nerve root visibility to guide injections. Our method of image quality assessment was conservative and accounted for the fact that some proceduralists still prefer to use visualization of the nerve root to guide needle placement. Based on the results of our study, we recommend a tube current of $40 \mathrm{~mA}$ or less for a body AP diameter of $\leq 30 \mathrm{~cm}$. This technique of stratifying patients by body size could also be used for selecting tube current for the planning CT, as prior studies have shown that the greatest contributor to dose for CT-guided lumbar injections is the planning CT. ${ }^{5}$

There are several limitations to this study. First, this is a retrospective study of a small number of patients at a single academic institution. The study included only 4 proceduralists, but we were able to select proceduralists with varying experience. The retrospective methodology results in a patient cohort with multiple and nonuniform combinations of tube current and body size. From this, we have derived a cutoff AP dimension for use of lower dose fluoroscopy, but a prospective study is required to validate the cutoff body size and better define the lower limit for tube current. Second, we did not study any clinical pain parameters. We felt that pain relief is multifactorial rather than solely correlated with technical success. Finally, we could not assess the rate of failed procedures due to poor image quality because all cases were technically successful in our study - in no case was the procedure stopped because of inability to precisely position the needle.

\section{Conclusions}

There is wide variability in tube current selection between proceduralists, despite little difference in patient body size between proceduralists. This calls for a more objective method of selecting tube current so that radiation dose can be minimized. Body size, measured by AP diameter on the scout image at the injection level, had the greatest influence on image quality. Patients with an AP diameter of $30 \mathrm{~cm}$ or less should be considered for CT fluoroscopy with lower tube current. 


\section{References}

1. Froelich JJ, Wagner HJ. CT-fluoroscopy: tool or gimmick? Cardiovasc Intervent Radiol 2001;24:297-305

2. Wagner AL. CT fluoroscopy-guided epidural injections: technique and results. AJNR Am J Neuroradiol 2004;25:1821-23

3. Meleka S, Patra A, Minkoff E, et al. Value of CT fluoroscopy for lumbar facet blocks. AJNR Am J Neuroradiol 2005;26:1001-03

4. Wagner AL. Selective lumbar nerve root blocks with CT fluoroscopic guidance: technique, results, procedure time, and radiation dose. AJNR Am J Neuroradiol 2004;25:1592-94

5. Hoang JK, Yoshizumi TT, Toncheva G, et al. Radiation dose exposure for lumbar spine epidural steroid injections: a comparison of conventional fluoroscopy data and CT fluoroscopy techniques. AJR Am J Roentgenol 2011;197:778-82

6. Aguirre DA, Bermudez S, Diaz OM. Spinal CT-guided interventional procedures for management of chronic back pain. J Vasc Interv Radiol 2005;16:689-97

7. Shepherd TM, Hess CP, Chin CT, et al. Reducing patient radiation dose during CT-guided procedures: demonstration in spinal injections for pain. AJNR Am J Neuroradiol 2011;32:1776-82

8. Young IA, Hyman GS, Packia-Raj LN, et al. The use of lumbar epidural/trans- foraminal steroids for managing spinal disease. J Am Acad Orthop Surg 2007; 15:228-38

9. Donnelly LF, Emery KH, Brody AS, et al. Minimizing radiation dose for pediatric body applications of single-detector helical CT: strategies at a large children's hospital. AJR Am J Roentgenol 2001;176:303-06

10. Zhou $Y$, Singh N, Abdi S, et al. Fluoroscopy radiation safety for spine interventional pain procedures in university teaching hospitals. Pain Physician 2005;8:49-53

11. Li X, Samei E, Segars WP, et al. Patient-specific dose estimation for pediatric chest CT. Med Phys 2008;35:5821-28

12. Singh S, Kalra MK, Moore MA, et al. Dose reduction and compliance with pediatric CT protocols adapted to patient size, clinical indication, and number of prior studies. Radiology 2009;252:200-08

13. Haaga JR, Miraldi F, MacIntyre W, et al. The effect of mAs variation upon computed tomography image quality as evaluated by in vivo and in vitro studies. Radiology 1981;138:449-54

14. Hoang JK, Gafton AR, Eastwood JD, et al. Correlation of cross-sectional diameter with image quality and radiation exposure in MDCT examinations of the neck. AJR Am J Roentgenol 2011:197:W904-09 